\title{
Survival of molecular gas in Virgo's hot intracluster medium: CO near M 86
}

\author{
K. M. Dasyra ${ }^{1}$, F. Combes ${ }^{1}$, P. Salomé ${ }^{1}$, and J. Braine ${ }^{2,3}$ \\ 1 Observatoire de Paris, LERMA, CNRS, 61 avenue de l'Observatoire, 75014 Paris, France \\ e-mail: kalliopi . dasyra@obspm.fr \\ 2 Univ. Bordeaux, Laboratoire d'Astrophysique de Bordeaux, UMR 5804, 33270 Floirac, France \\ 3 CNRS, LAB, UMR 5804, 33270 Floirac, France
}

Received 4 January 2012 / Accepted 9 February 2012

\section{ABSTRACT}

\begin{abstract}
We carried out ${ }^{12} \mathrm{CO}(1-0)$ and ${ }^{12} \mathrm{CO}(2-1)$ observations of 21 different regions in the vicinity of $\mathrm{M} 86$, NGC 4438 , and along the $120 \mathrm{kpc}-$ long, $\mathrm{H} \alpha$-emitting filamentary trail that connects them, aiming to test whether molecular gas can survive to be transferred from a spiral to an elliptical galaxy in Virgo's $10^{7} \mathrm{~K}$ intracluster medium (ICM). We targeted H $\alpha$-emitting regions that could be associated with the interface between cold molecular clouds and the hot ionized ICM. The data, obtained with the $30 \mathrm{~m}$ telescope of the Institut de Radioastronomie Millimétrique, led to the detection of molecular gas close to M 86. CO gas with a recession velocity that is similar to that of the stars, $-265 \mathrm{~km} \mathrm{~s}^{-1}$, and with a corresponding $\mathrm{H}_{2}$ mass of $2 \times 10^{7} M_{\odot}$, was detected $\sim 10 \mathrm{kpc}$ southeast of the nucleus of M 86, near the peak of its $\mathrm{H}$ I emission. We argue that it is possible for this molecular gas either to have formed in situ from $\mathrm{H}$, or to have been stripped from NGC 4438 directly in molecular form. In situ formation is nonetheless negligible for the $7 \times 10^{6} M_{\odot}$ of gas detected at 12:26:15.9+12:58:49, at $\sim 10 \mathrm{kpc}$ northeast of M 86, where no (strong) $\mathrm{H}_{\mathrm{I}}$ emission is present. This detection provides evidence for the survival of molecular gas in filaments for timescales of $\sim 100$ Myr. An amount equivalent to $5 \times 10^{7} M_{\odot}$ of $\mathrm{H}_{2}$ gas that could be lost to the ICM or to neighboring galaxies was also discovered in the tidal tail northwest of NGC 4438. A scenario in which gas was alternatively brought to M 86 from NGC 4388 was also examined but it was considered unlikely because of the non-detection of CO below or at the H I stream velocities, 2000-2700 $\mathrm{km} \mathrm{s}^{-1}$.
\end{abstract}

Key words. evolution - ISM: kinematics and dynamics - galaxies: interactions - galaxies: clusters: individual: Virgo galaxies: clusters: intracluster medium - ISM: clouds

\section{Introduction}

Stripping of gas during galaxy collisions can replenish the gas reservoirs of elliptical galaxies and reinitiate star formation in them, in particular in overdense cluster environments. Cooling flows can further add to the deposition of external gas in cluster ellipticals (see Fabian 1994, and references therein). The growth of these ellipticals is regulated by the interplay of gas cooling and heating along gas filaments that are associated with either mergers or cooling flows and that have often been discovered in the $\mathrm{H} \alpha$ and $\left[\mathrm{N}_{\mathrm{II}}\right.$ ] images of local clusters (Cowie et al. 1983; Heckman et al. 1989; Conselice et al. 2001; Kenney et al. 2008). This interplay manifests itself and can be examined through the detection of multi-phase gas emission lines in the same clouds. Indeed, warm atomic gas, warm molecular gas, and cold molecular gas clouds have been found to coexist in spatially resolved filaments around NGC 1275 (Perseus A), where they were detected through $\mathrm{H} \alpha, \mathrm{H}_{2}$, and $\mathrm{CO}$ emission (Conselice et al. 2001; Salomé et al. 2006, 2011; Johnstone et al. 2007).

Several processes, however, occur in cluster environments that can impede the transfer of gas from one galaxy to another. Ram pressure stripping (Gunn \& Gott 1972), for example, can disperse the diffuse atomic gas into the intracluster medium (ICM). Even though the ram pressure drag is negligible for the motions of the dense molecular gas clouds (Nulsen 1982; Kenney \& Young 1989), it can eventually destroy them by depleting the atomic gas reservoir from which they reform (Crowl et al. 2005). The molecular clouds are also several orders of magnitude cooler than their environment and can be easily destroyed by X-rays if they are not self-shielded. The ICM X-ray spectrum often peaks in the range $0.7-2.0 \mathrm{keV}$, indicating temperatures of $\sim 10^{7} \mathrm{~K}$ (e.g., Rangarajan et al. 1995; Machacek et al. 2004; Fabian et al. 2006; Randall et al. 2008; Tamura et al. 2009).

Evidence that molecular clouds do nonetheless survive or reform in $\gtrsim 10^{7} \mathrm{~K}$ intracluster and intragalactic media has been found. Braine et al. (2000) discovered CO in a tidal tail dwarf galaxy of Arp 105, in the X-ray emitting medium of Abell 1185 (Mahdavi et al. 1996). Because the CO-emitting regions spatially coincided and had comparable kinematic properties with the H I-emitting regions, Braine et al. (2000) proposed in situ formation of molecules. In addition to the molecular gas detected in the cooling-flow-related filaments around NGC 1275 in Perseus (Salomé et al. 2006, 2011; Johnstone et al. 2007), Vollmer et al. (2008) found extraplanar CO near NGC 4522 in Virgo, in regions where atomic gas was brought by ram pressure. In ESO137-001, located in Abell 3627, a $\sim 10^{7} \mathrm{~K}$ ram-pressuredriven gas tail has active star formation and $\mathrm{H}_{2}$ emission (Sun et al. 2010; Sivanandam et al. 2010). In the compact group of Stephan's Quintet, $\mathrm{H}_{2}$ molecules not only survive in a medium of comparable temperature (Trinchieri et al. 2005; O'Sullivan et al. 2009), but they are mainly responsible for the gas cooling instead of the X-rays (Cluver et al. 2010).

An excellent system to test whether molecular gas could be directly brought to an elliptical galaxy, under extreme conditions, from spiral(s) interacting with it is in the Virgo cluster. 


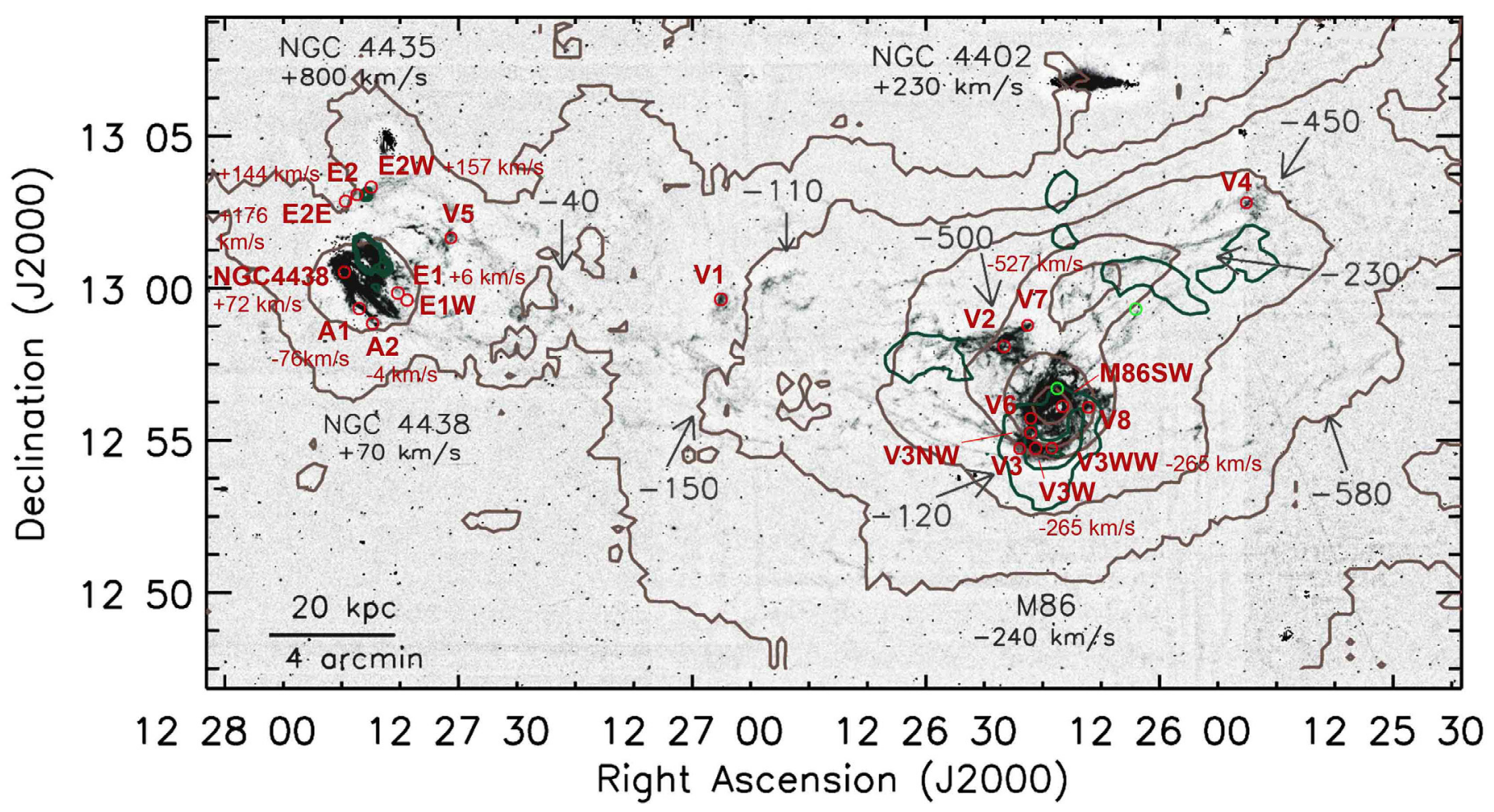

Fig. 1. Finding chart of our observations. The positions of previous CO observations (Wiklind et al. 1995; Braine et al. 1997) are marked with light green circles. HI line contours (dark green) and X-ray contours (brown) are plotted over the narrow band H $\alpha+[\mathrm{N}$ II] image of Virgo's core (adapted from Kenney et al. 2008). The velocities appearing in gray correspond to those of the $\mathrm{H} \alpha$ gas as measured by the same authors. In red, we mark the $\mathrm{H} \alpha$ clouds that we observed searching for associated $\mathrm{CO}$ emission. Upon detection, we also indicate the velocity of the molecular gas traced by the ${ }^{12} \mathrm{CO}(1-0)$ line. In addition to these 20 regions, we also observed at 12:27:30.0+13:12:12.0, northwest of NGC 4435 , in the clouds that Cortese et al. (2010a) denote as plume.

The dust clumps that are detected in the giant elliptical galaxy M 86 (also known as NGC 4406) could have originated from its past interaction with its neighboring spiral galaxy NGC 4438 (Cortese et al. 2010b; Gomez et al. 2010). Dust and gas could have been captured through ram pressure stripping of the interstellar medium (ISM) of NGC 4438 by that of M 86 (Kenney et al. 2008), through dynamical instabilities, or through both processes. The H I content deficit of NGC 4438 relative to its own $\mathrm{CO}$ emission confirms that ram pressure has acted on its ISM (Vollmer et al. 2005, 2009; Hota et al. 2007). Dynamical perturbations continue to distort NGC 4438, as indicated by the presence of stars and gas in its northern tidal tail toward NGC 4435 (Combes et al. 1988). The interaction between NGC 4438 and M 86 is confirmed by a spectacular, $120 \mathrm{kpc}-$ long, $\mathrm{H} \alpha$-emitting tidal bridge that connects them (Kenney et al. 2008). The smooth $\mathrm{H} \alpha$ velocity gradient along this bridge convincingly places it to the ICM of Virgo, which is rich in X-ray emission (Forman et al. 1979; Rangarajan et al. 1995; Machacek et al. 2004; Liu \& Bregman 2005; Randall et al. 2008). Since the X-ray heating and the ram pressure disperse the ICM atomic gas, a significant fraction of the $\mathrm{H} \alpha$ emission could originate from the ionized gas in the outer layers of molecular clouds (Ferland et al. 2009). This makes the H $\alpha$ clumps in the NGC 4438-M 86 bridge prime targets for testing whether star formation can be initiated from gas that has either been brought from NGC 4438 to M 86 in the molecular state, or from molecules that recombined in situ in M 86 after being transferred into atomic state.

We obtained mm data with the $30 \mathrm{~m}$ telescope of the Institut de Radioastronomie Millimétrique (IRAM) to test for the presence of molecular gas in some of the $\mathrm{H} \alpha$ filaments that are embedded in Virgo's hot ICM. We focused on $\mathrm{H} \alpha$ emission peaks, aiming to determine whether the warm atomic gas emission could be associated with cold gas clouds, to compute the amount of molecular gas and potential star formation rate (SFR) in $\mathrm{M} \mathrm{86}$, and to test the gas origin by examining whether the molecules could have been transferred there or formed in situ. We assume a distance of $17.5 \mathrm{Mpc}$ to Virgo throughout this work (Mei et al. 2007).

\section{Observations and data analysis}

The observations were carried out with the $30 \mathrm{~m}$ telescope at Pico Veleta, Spain. We observed twenty $\mathrm{H} \alpha$-emitting regions in or near NGC 4438 and M 86, and along the tidal bridge that connects them (Fig. 1), to test whether they contain detectable amounts of $\mathrm{CO}$. In addition to these positions, we also observed clouds in the diffuse, X-ray emitting plume northwest of NGC 4438 and NGC 4435, which is most likely attributed to Galactic cirrus (Cortese et al. 2010a).

We simultaneously observed the ${ }^{12} \mathrm{CO} J=1-0$ and $J=$ 2-1 lines with the EMIR receivers. The receivers were tuned to the frequencies of the two transitions at $115.271 \mathrm{GHz}$ and $230.538 \mathrm{GHz}$, respectively, shifted to the velocity of the $\mathrm{H} \alpha$ gas in each position. All of the $4 \mathrm{MHz}$, WILMA, and VESPA backends, with corresponding resolutions of $4 \mathrm{MHz}, 2 \mathrm{MHz}$, and $0.3 \mathrm{MHz}$, were simultaneously attached to allow consistency checks, with the WILMA data being primarily used in this work. VESPA data are only shown for the plume. The observations were performed in wobbler-switching mode with a throw of 2!.40. The telescope pointing was monitored by observing a bright radio source every two hours, indicating a pointing accuracy of $2^{\prime \prime}$ to $3^{\prime \prime}$. The system temperature varied from 270 
K. M. Dasyra et al.: CO in Virgo's hot intracluster medium
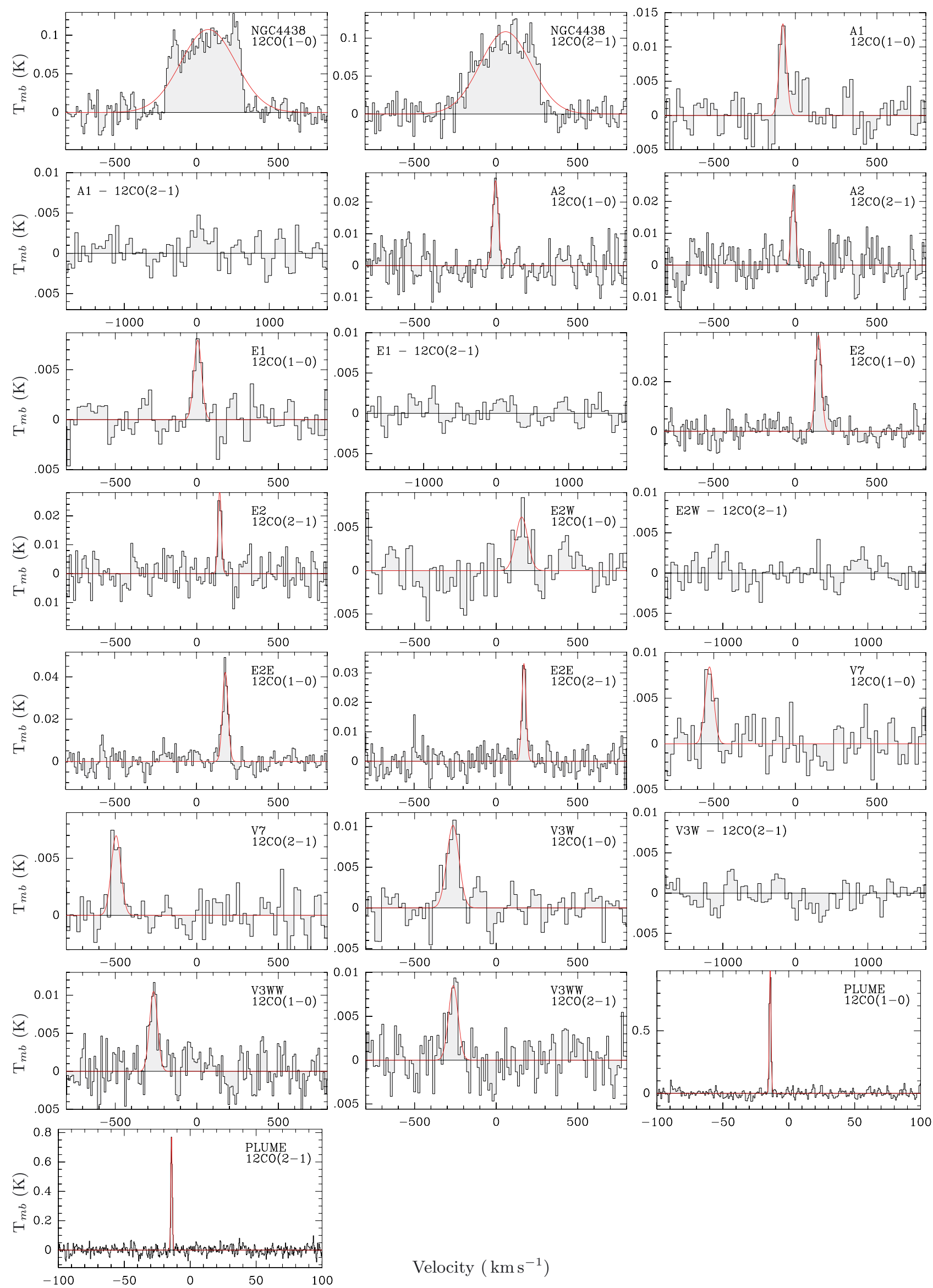

Velocity $\left(\mathrm{km} \mathrm{s}^{-1}\right)$

Fig. 2. Spectra of the plume and of the Virgo regions shown in Fig. 1 with ${ }^{12} \mathrm{CO}(1-0)$ line detections. Gaussian fits to the $\mathrm{CO}$ line profiles (black) are overplotted in red. 
Table 1. ${ }^{12} \mathrm{CO}(1-0)$ and ${ }^{12} \mathrm{CO}(2-1)$ line properties and derived gas masses in different Virgo regions.

\begin{tabular}{|c|c|c|c|c|c|c|c|c|c|}
\hline Position & $\begin{array}{c}\text { Coordinates } \\
(\mathrm{J} 2000)\end{array}$ & $\begin{array}{l}\text { Integration } \\
\text { time (min) }\end{array}$ & Line & $\begin{array}{c}S(\mathrm{CO})^{a} \\
\left(\mathrm{Jy} \mathrm{km} \mathrm{s}^{-1}\right)\end{array}$ & $\begin{array}{c}V^{b} \\
\left(\mathrm{~km} \mathrm{~s}^{-1}\right)\end{array}$ & $\begin{array}{c}F W H M^{c} \\
\left(\mathrm{~km} \mathrm{~s}^{-1}\right)\end{array}$ & $\begin{array}{l}T_{\mathrm{mb}}{ }^{d} \\
(\mathrm{mK})\end{array}$ & $\begin{array}{c}\text { Resolution } \\
\left(\mathrm{km} \mathrm{s}^{-1}\right)\end{array}$ & $\begin{array}{l}M_{\text {gas }}^{e} \\
\left(M_{\odot}\right)\end{array}$ \\
\hline NGC 4438 & $12: 27: 45.60+13: 00: 32.0$ & 15 & ${ }^{12} \mathrm{CO}(1-0)$ & $226 \pm 6$ & $72 \pm 6$ & $395 \pm 12$ & $108 \pm 14$ & 10 & $5.7 \times 10^{8}$ \\
\hline NGC 4438 & & 15 & ${ }^{12} \mathrm{CO}(2-1)$ & $208 \pm 8$ & $61 \pm 7$ & $362 \pm 14$ & $108 \pm 15$ & 13 & $\ldots$ \\
\hline A1 & $12: 27: 43.70+12: 59: 22.8$ & 110 & ${ }^{12} \mathrm{CO}(1-0)$ & $3.5 \pm 0.6$ & $-76 \pm 5$ & $46 \pm 11$ & $13.4 \pm 3$ & 21 & $8.8 \times 10^{6}$ \\
\hline A1 & & 109 & ${ }^{12} \mathrm{CO}(2-1)$ & $\ldots$ & $\ldots$ & $\ldots$ & $<5.1$ & 47 & $\ldots$ \\
\hline A2 & $12: 27: 42.10+12: 58: 51.4$ & 25 & ${ }^{12} \mathrm{CO}(1-0)$ & $5.4 \pm 0.6$ & $-4 \pm 2$ & $37 \pm 5$ & $27 \pm 5$ & 10 & $1.4 \times 10^{7}$ \\
\hline A2 & & 25 & ${ }^{12} \mathrm{CO}(2-1)$ & $3.8 \pm 0.6$ & $-12 \pm 3$ & $29 \pm 6$ & $24 \pm 5$ & 10 & $\ldots$ \\
\hline E1 & $12: 27: 38.60+12: 59: 52.0$ & 90 & ${ }^{12} \mathrm{CO}(1-0)$ & $2.3 \pm 0.4$ & $6 \pm 5$ & $52 \pm 12$ & $7.9 \pm 1.6$ & 21 & $5.8 \times 10^{6}$ \\
\hline E1 & & 90 & ${ }^{12} \mathrm{CO}(2-1)$ & $\ldots$ & $\ldots$ & $\ldots$ & $<3.8$ & 47 & $\ldots$ \\
\hline E1W & $12: 27: 37.40+12: 59: 38.3$ & 37 & ${ }^{12} \mathrm{CO}(1-0)$ & $\ldots$ & $\ldots$ & $\ldots$ & $<5.4$ & 47 & $<3.2 \times 10^{6}$ \\
\hline E1W & & 37 & ${ }^{12} \mathrm{CO}(2-1)$ & $\ldots$ & $\ldots$ & $\ldots$ & $<3.9$ & 47 & $\ldots$ \\
\hline E2 & $12: 27: 44.00+13: 03: 00.0$ & 50 & ${ }^{12} \mathrm{CO}(1-0)$ & $8.8 \pm 0.7$ & $144 \pm 2$ & $42 \pm 5$ & $39 \pm 3$ & 10 & $2.2 \times 10^{7}$ \\
\hline E2 & & 50 & ${ }^{12} \mathrm{CO}(2-1)$ & $3.8 \pm 0.7$ & $141 \pm 2$ & $21 \pm 6$ & $29 \pm 5$ & 13 & $\ldots$ \\
\hline E2E & $12: 27: 45.50+13: 02: 48.0$ & 65 & ${ }^{12} \mathrm{CO}(1-0)$ & $8.7 \pm 0.6$ & $176 \pm 1$ & $38 \pm 3$ & $42 \pm 4$ & 10 & $2.2 \times 10^{7}$ \\
\hline E2E & & 65 & ${ }^{12} \mathrm{CO}(2-1)$ & $5.3 \pm 0.6$ & $172 \pm 2$ & $28 \pm 5$ & $33 \pm 4$ & 10 & $\ldots$ \\
\hline E2W & $12: 27: 42.10+13: 03: 15.0$ & 80 & ${ }^{12} \mathrm{CO}(1-0)$ & $2.6 \pm 0.6$ & $157 \pm 10$ & $84 \pm 22$ & $6.1 \pm 2.1$ & 21 & $6.5 \times 10^{6}$ \\
\hline E2W & & 80 & ${ }^{12} \mathrm{CO}(2-1)$ & $\ldots$ & $\ldots$ & $\ldots$ & $<4.8$ & 47 & $\ldots$ \\
\hline V5 & $12: 27: 31.60+13: 01: 38.0$ & 42 & ${ }^{12} \mathrm{CO}(1-0)$ & $\ldots$ & $\ldots$ & $\ldots$ & $<5.4$ & 47 & $<3.2 \times 10^{6}$ \\
\hline V5 & & 42 & ${ }^{12} \mathrm{CO}(2-1)$ & $\ldots$ & $\ldots$ & $\ldots$ & $<3.8$ & 47 & $\ldots$ \\
\hline V1 & $12: 26: 56.20+12: 59: 40.0$ & 52 & ${ }^{12} \mathrm{CO}(1-0)$ & $\ldots$ & $\ldots$ & $\ldots$ & $<4.7$ & 47 & $<2.8 \times 10^{6}$ \\
\hline V1 & & 52 & ${ }^{12} \mathrm{CO}(2-1)$ & $\ldots$ & $\ldots$ & $\ldots$ & $<6.4$ & 47 & $\ldots$ \\
\hline V2 & $12: 26: 19.00+12: 58: 10.0$ & 43 & ${ }^{12} \mathrm{CO}(1-0)$ & $\ldots$ & $\ldots$ & $\ldots$ & $<4.7$ & 47 & $<2.8 \times 10^{6}$ \\
\hline $\mathrm{V} 2$ & & 43 & ${ }^{12} \mathrm{CO}(2-1)$ & $\ldots$ & $\ldots$ & $\ldots$ & $<4.1$ & 47 & $\ldots$ \\
\hline V7 & $12: 26: 15.90+12: 58: 49.0$ & 50 & ${ }^{12} \mathrm{CO}(1-0)$ & $2.8 \pm 0.5$ & $-527 \pm 6$ & $59 \pm 12$ & $8.4 \pm 1.9$ & 21 & $7.1 \times 10^{6}$ \\
\hline V7 & & 45 & ${ }^{12} \mathrm{CO}(2-1)$ & $2.6 \pm 0.4$ & $-495 \pm 6$ & $66 \pm 13$ & $7.0 \pm 1.4$ & 21 & $\ldots$ \\
\hline V4 & $12: 25: 47.50+13: 02: 44.0$ & 23 & ${ }^{12} \mathrm{CO}(1-0)$ & $\ldots$ & $\ldots$ & $\ldots$ & $<6.5$ & 47 & $<3.9 \times 10^{6}$ \\
\hline V4 & & 23 & ${ }^{12} \mathrm{CO}(2-1)$ & $\ldots$ & $\ldots$ & $\ldots$ & $<4.4$ & 47 & $\ldots$ \\
\hline V3 & $12: 26: 17.00+12: 54: 54.0$ & 37 & ${ }^{12} \mathrm{CO}(1-0)$ & $\ldots$ & $\ldots$ & $\ldots$ & $<5.3$ & 47 & $<3.1 \times 10^{6}$ \\
\hline V3 & & 37 & ${ }^{12} \mathrm{CO}(2-1)$ & $\ldots$ & $\ldots$ & $\ldots$ & $<5.6$ & 47 & $\ldots$ \\
\hline V3W & $12: 26: 14.90+12: 54: 54.0$ & 129 & ${ }^{12} \mathrm{CO}(1-0)$ & $4.9 \pm 0.6$ & $-265 \pm 6$ & $89 \pm 15$ & $10 \pm 2$ & 21 & $1.2 \times 10^{7}$ \\
\hline V3W & & 129 & ${ }^{12} \mathrm{CO}(2-1)$ & $\ldots$ & $\ldots$ & $\ldots$ & $<4.4$ & 47 & $\ldots$ \\
\hline V3WW & $12: 26: 12.80+12: 54: 54.0$ & 115 & ${ }^{12} \mathrm{CO}(1-0)$ & $3.2 \pm 0.4$ & $-265 \pm 4$ & $57 \pm 9$ & $11 \pm 2.3$ & 10 & $8.1 \times 10^{6}$ \\
\hline V3WW & & 115 & ${ }^{12} \mathrm{CO}(2-1)$ & $3.1 \pm 0.5$ & $-263 \pm 6$ & $68 \pm 16$ & $8.5 \pm 2.2$ & 13 & $\ldots$ \\
\hline V3NW & $12: 26: 15.50+12: 55: 22.0$ & 40 & ${ }^{12} \mathrm{CO}(1-0)$ & $\ldots$ & $\ldots$ & $\ldots$ & $<8.0$ & 47 & $<4.7 \times 10^{6}$ \\
\hline V3NW & & 40 & ${ }^{12} \mathrm{CO}(2-1)$ & $\ldots$ & $\ldots$ & $\ldots$ & $<5.5$ & 47 & $\ldots$ \\
\hline V6 & $12: 26: 15.50+12: 55.50 .0$ & 35 & ${ }^{12} \mathrm{CO}(1-0)$ & $\ldots$ & $\ldots$ & $\ldots$ & $<7.5$ & 47 & $<4.4 \times 10^{6}$ \\
\hline V6 & & 35 & ${ }^{12} \mathrm{CO}(2-1)$ & $\ldots$ & $\ldots$ & $\ldots$ & $<4.5$ & 47 & $\ldots$ \\
\hline V8 & $12: 26: 08.00+12: 56: 12.0$ & 35 & ${ }^{12} \mathrm{CO}(1-0)$ & $\ldots$ & $\ldots$ & $\ldots$ & $<7.3$ & 47 & $<4.3 \times 10^{6}$ \\
\hline V8 & & 35 & ${ }^{12} \mathrm{CO}(2-1)$ & $\ldots$ & $\ldots$ & $\ldots$ & $<6.5$ & 47 & $\ldots$ \\
\hline M86SW & $12: 26: 11.40+12: 56: 15.0$ & 23 & ${ }^{12} \mathrm{CO}(1-0)$ & $\ldots$ & $\ldots$ & $\ldots$ & $<12$ & 47 & $<7.1 \times 10^{6}$ \\
\hline M86SW & & 23 & ${ }^{12} \mathrm{CO}(2-1)$ & $\ldots$ & $\ldots$ & $\ldots$ & $<12$ & 47 & $\ldots$ \\
\hline PLUME & $12: 27: 30.00+13: 12: 12.0$ & 10 & ${ }^{12} \mathrm{CO}(1-0)$ & $8.1 \pm 0.2$ & -14 & 1.2 & $1018 \pm 27$ & 0.8 & $\ldots$ \\
\hline PLUME & & 10 & ${ }^{12} \mathrm{CO}(2-1)$ & $5.2 \pm 0.1$ & -14 & 1.2 & $782 \pm 27$ & 0.4 & $\ldots$ \\
\hline
\end{tabular}

Notes. ${ }^{(a)}$ Fluxes obtained from the Gaussian fitting to the line profiles, and multiplied by a temperature-to-flux conversion factor of $5.0 \mathrm{Jy} / \mathrm{K}$ that is appropriate for the $30 \mathrm{~m}$ telescope at all observed frequencies. ${ }^{(b)}$ All velocities are heliocentric. ${ }^{(c)}$ Resolution-corrected line width. ${ }^{(d)}$ All limits are computed at $3 \sigma$ levels. ${ }^{(e)}$ A standard CO-to- $\mathrm{H}_{2}$ Galactic value of $4.6 M_{\odot}\left(\mathrm{K} \mathrm{km} \mathrm{s}^{-1} \mathrm{pc}^{2}\right)^{-1}$ was used for the mass computation. The $3 \sigma$ limits were computed for a width of $47 \mathrm{~km} \mathrm{~s}^{-1}$. This is close to the median ${ }^{12} \mathrm{CO}(1-0)$ width for all regions but the plume and NGC 4438 .

to $500 \mathrm{~K}$ at $3 \mathrm{~mm}$. We typically spent $0.25-2 \mathrm{~h}$ on source, which led to $1 \sigma$ noise levels of $2 \mathrm{mK}$ to $10 \mathrm{mK}$ at the frequency of the ${ }^{12} \mathrm{CO}(1-0)$ transition (Table 1$)$.

The data were reduced with the CLASS software of IRAM. We averaged the spectra of each region after removing bad channels and subtracting linear baselines, dropping scans with baseline fluctuations. The resulting spectra, binned to $10-21 \mathrm{~km} \mathrm{~s}^{-1}$ channels, are shown in Fig. 2. For the plume, the high-resolution VESPA spectra are unbinned. A Gaussian function was fitted to each line for the derivation of its width, peak position, temperature $T$, and flux $S$, which are all shown in Table 1 . Assuming that the observed clouds are unresolved, $T$ corresponds to the main beam temperature. It was converted to a flux using a temperature-to-flux conversion factor of 5.0 Jy/K (appropriate for both the 1 and $3 \mathrm{~mm}$ observations).

The cold $\mathrm{H}_{2}$ gas masses, which are also presented in Table 1, were computed as

$$
M_{\mathrm{H}_{2}}=\alpha \frac{23.5 I_{\mathrm{CO}} \Omega_{\mathrm{B}} D_{\mathrm{L}}^{2}}{(1+z)^{3}} M_{\odot} \text {, }
$$




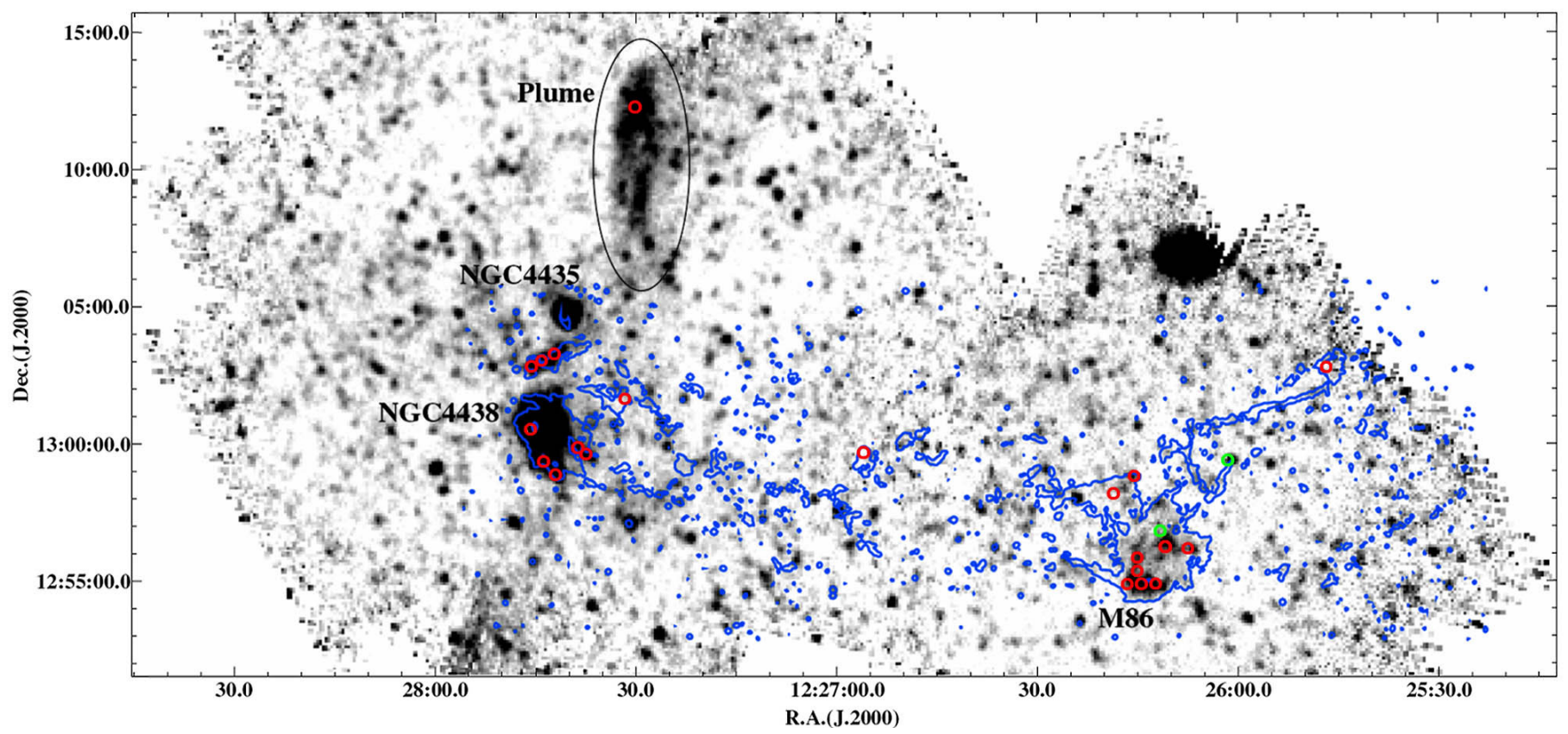

Fig. 3. Our CO observation positions (red circles) and positions of previous CO observations (Wiklind et al. 1995; Braine et al. 1997; green circles), over Herschel $250 \mu \mathrm{m}$ SPIRE imaging data (adapted from Cortese et al. 2010b). The H $\alpha$ intensity contours are overplotted in blue.

where $I_{\mathrm{CO}}$ is the $115 \mathrm{GHz}$ line intensity in $\mathrm{K} \mathrm{km} \mathrm{s}^{-1}, \Omega_{\mathrm{B}}$ is the main beam area in $\operatorname{arcsec}^{2}$ (for unresolved clouds), $D_{\mathrm{L}}$ is the luminosity distance in $\mathrm{Mpc}$, and $\alpha$ is the $\mathrm{CO}$ luminosity to $\mathrm{H}_{2}$ mass conversion factor (Solomon et al. 1997). We used a standard Galactic conversion factor of $4.6 M_{\odot}\left(\mathrm{K} \mathrm{km} \mathrm{s}^{-1} \mathrm{pc}^{2}\right)^{-1}$ (Bloemen et al. 1986; Downes et al. 1993), and a telescope beam of $22^{\prime \prime}$ (i.e., $1.8 \mathrm{kpc}$ ) at $3 \mathrm{~mm}$. The $3 \sigma$ mass limits for non-detections were computed using a resolution (and width) of $47 \mathrm{~km} \mathrm{~s}^{-1}$, which is characteristic of the clouds under examination (see Table 1).

\section{Results: CO-emitting vs. non-emitting regions}

Narrow ${ }^{12} \mathrm{CO}(1-0)$ and ${ }^{12} \mathrm{CO}(2-1)$ emission is found in the plume clouds northwest of NGC 4438 and NGC 4435, at $12: 27: 30+13: 12: 12$. Both line widths are of only $1.2 \mathrm{~km} \mathrm{~s}^{-1}$, confirming that the emission originates from Galactic cirrus clouds. This possibility was suggested by Cortese et al. (2010a) based on the ${ }^{12} \mathrm{CO}(1-0)$ line width at a nearby position, $12: 27: 30+13: 12: 29$. CO lines were detected in another 10 of the 20 regions shown in Fig. 1, but their kinematics were atypical of cirrus clouds, with line width(s) exceeding $30 \mathrm{~km} \mathrm{~s}^{-1}$ in all cases. A cross examination of the high-resolution VESPA data confirms this finding.

Several regions in Virgo, at $\sim 10 \mathrm{kpc}$ from the $B$-band center of M 86 (at 12:26:12+12:56:49) had a CO detection. The region V7, which is located $11 \mathrm{kpc}$ northeast of M 86 and in the northwest tip of the local $\mathrm{H} \alpha$ filamentary structure (Fig. 1), contains $7.1 \times 10^{6} M_{\odot}$ of gas that is moving at $-527 \mathrm{~km} \mathrm{~s}^{-1}$. This is very close to the measured $\mathrm{H} \alpha$ recession velocity, $-500 \mathrm{~km} \mathrm{~s}^{-1}$ (Kenney et al. 2008). This line-of-sight velocity precludes a clear answer to the question of whether or not the clouds are bound to $\mathrm{M} 86$. The escape velocity at a distance of $10 \mathrm{kpc}$ from the center of M 86 is high, $470 \mathrm{~km} \mathrm{~s}^{-1}$, because its bulge mass equals $2.6 \times 10^{11} M_{\odot}$. The latter is given in units of $10^{10} M_{\odot}$ by $c_{2} \sigma_{100}{ }^{2} R_{\text {eff }}$, where $c_{2}$ is a unitless coefficient depending on the matter distribution, $\sigma_{100}$ is the stellar velocity dispersion in units of $100 \mathrm{~km} \mathrm{~s}^{-1}$, and $R_{\mathrm{eff}}$ is the effective radius in $\mathrm{kpc}$. The value of $c_{2}$ for giant ellipticals is 1 (Bender et al. 1992). For M 86, $\sigma_{100}$ is 2.2 (Smith et al. 2000) and $R_{\text {eff }}$ is $5.5 \mathrm{kpc}$ (Gavazzi et al. 2005) in the $B$ band.

$\mathrm{CO}$ gas was also found in the $\mathrm{V} 3 \mathrm{~W}$ and $\mathrm{V} 3 \mathrm{WW}$ regions, along an $\mathrm{H} \alpha$ stream that results in the center of $\mathrm{M} 86$, at 9 to $10 \mathrm{kpc}$ away from it (Fig. 1). The $\mathrm{CO}$ emission coincides with the H I emission peak of M 86 (Li \& van Gorkom 2001; Kenney et al. 2008). The recession velocity of the CO, $-265 \mathrm{~km} \mathrm{~s}^{-1}$, agrees well with that measured from the $\mathrm{HI},-240 \mathrm{~km} \mathrm{~s}^{-1}$ (Bregman \& Roberts 1990), and from the Mg stellar absorption features in optical wavelengths, $-244 \mathrm{~km} \mathrm{~s}^{-1}$ (Smith et al. 2000). The ${ }^{12} \mathrm{CO}(1-0)$ FWHM in these regions, $50-90 \mathrm{~km} \mathrm{~s}^{-1}$, is comparable with the narrow H I component FHWM, $60 \mathrm{~km} \mathrm{~s}^{-1}$ (Bregman \& Roberts 1990). The combined $\mathrm{H}_{2}$ mass for both $\mathrm{V} 3 \mathrm{~W}$ and $\mathrm{V} 3 \mathrm{WW}$ regions is $2.0 \times 10^{7} M_{\odot}$. If stars are forming out of this gas, and if the galaxy-integrated SFR calibration of Gao \& Solomon (2004) is applicable for these clouds, then the SFR will be $0.03 M_{\odot} \mathrm{yr}^{-1}$. Likewise, the combination of the modified black body fit to SPIRE data (Cortese et al. 2010b, Figs. 3 and 4) of the same regions that was performed by Gomez et al. (2010) and the Kennicutt (1998) relation lead to a dustbased SFR estimate that is also on the order of $0.01 M_{\odot} \mathrm{yr}^{-1}$.

No molecular gas was found at their neighboring V3, V3NW, V6, V8, and M86SW regions. The latter is along the same $\mathrm{H} \alpha$ stream connecting V3W and V3WW to the very center of M 86. For $T<12 \mathrm{mK}$ and for a line width of $47 \mathrm{~km} \mathrm{~s}^{-1}$, the gas mass in M86SW is $<7 \times 10^{6} M_{\odot}$. Molecular gas has not been detected at the center of M 86 either (Wiklind et al. 1995; Braine et al. 1997); if it exists, its mass must be below $5 \times 10^{6} M_{\odot}$ for the assumptions in this work.

No gas was found either at V1, V2, and V5 of the tidal bridge between NGC 4438 and M 86, or at V4, which is located in the figure-of-eight shaped $\mathrm{H} \alpha$ loop northwest of $\mathrm{M} 86^{1}$. If gas does exist in any of these regions, its mass is below $4 \times 10^{6} M_{\odot}$.

1 This loop belongs to Virgo's ICM and part of it could be bound to $\mathrm{M} 86$, because its $\mathrm{H} \alpha$ recession velocity ranges from -230 to $-450 \mathrm{~km} \mathrm{~s}^{-1}$. 


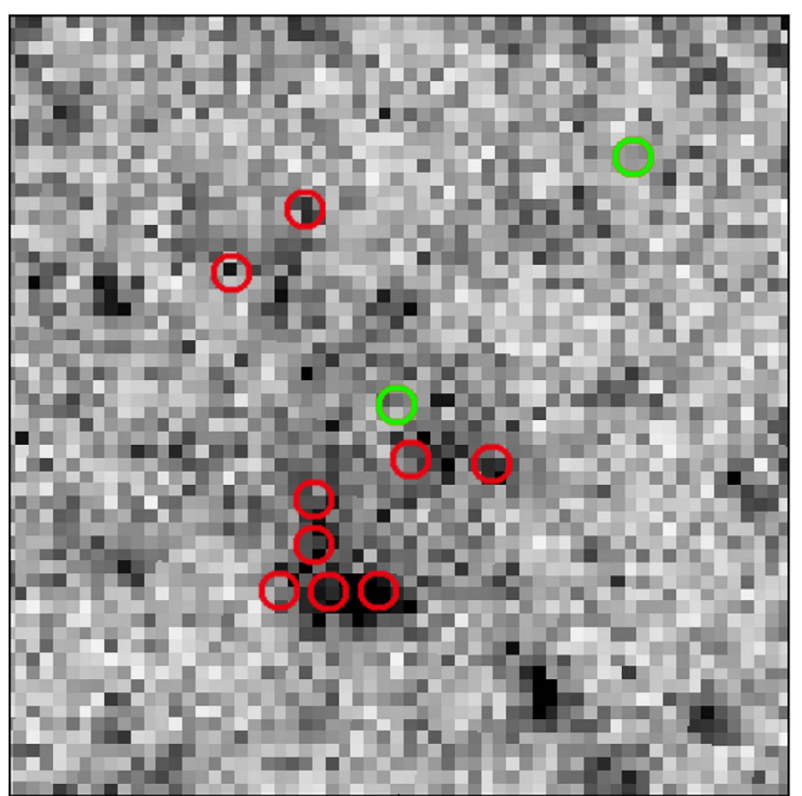

Fig. 4. Our observations plotted over a $4^{\prime} \times 4^{\prime}$ zoom around M 86 at $350 \mu \mathrm{m}$. The far-infrared image is taken from the first public data release of the Herschel Virgo Cluster Survey (HeVICS), which was initially presented in Davies et al. (2010). The CO positions are color-coded as in Fig. 3.

Previous observations by Wiklind et al. (1995) at another location of this loop, 12:26:02+12:59:21, also led to a non-detection, with an upper limit of $7.9 \times 10^{6} M_{\odot}$.

Most of the CO gas is detected in or around NGC 4438. At the center of NGC 4438, the telescope beam encompasses $6 \times 10^{8} M_{\odot}$ of $\mathrm{H}_{2}$ gas that has a recession velocity identical to that of the $\mathrm{H} \alpha$. The ${ }^{12} \mathrm{CO}(1-0)$ line profile also agrees well with that found from previous observations by Vollmer et al. (2005). Both the ${ }^{12} \mathrm{CO}(1-0)$ and the ${ }^{12} \mathrm{CO}(2-1)$ profiles indicate that the gas is in a disk whose rotational velocity reaches $200 \mathrm{~km} \mathrm{~s}^{-1}$ within the inner $4 \mathrm{kpc}$ of NGC $4438^{2}$. New observations are presented here for the gas clouds at $\mathrm{E} 1$, and in the regions E2, E2E, and E2W at $\sim 10 \mathrm{kpc}$ north of the nucleus. We specifically observed parts of the $\mathrm{H} \alpha$ filament that is oriented perpendicularly to the tidal tail northeast of NGC 4438, which was previously observed by Vollmer et al. (2005). The nearest positions of our observations and those of Vollmer et al. (2005) are one beam $\left(22^{\prime \prime}\right)$ away. The clouds in the E2 complexes are moving at +70 to $+100 \mathrm{~km} \mathrm{~s}^{-1}$ from systemic velocity along the line of sight. Their total $\mathrm{H}_{2}$ mass is $5.1 \times 10^{7} M_{\odot}$. Their counterparts in the south of the nucleus are named A1 and A2. They contain $2.3 \times 10^{7} M_{\odot}$ of $\mathrm{H}_{2}$ gas, and they are moving at -80 to $-150 \mathrm{~km} \mathrm{~s}^{-1}$ from systemic velocity. For both the northern and southern clouds, the observed velocities are consistent with gas that is either part of a rotating disk, or experiencing tidal and ram pressure stripping (Vollmer et al. 2005). If all of these A and E2 cloud complexes are stripped, a considerable (14\%) fraction of the gas in the center of NGC 4438 (see Table 1) is potentially lost to the ICM and to neighboring galaxies.

\footnotetext{
${ }^{2}$ The differences in the observed ${ }^{12} \mathrm{CO}(1-0)$ and ${ }^{12} \mathrm{CO}(2-1)$ line properties of other regions, such as E2W, E2, and E2E, can be accounted for by differences in the gas density and excitation properties, or differences in the sensitivity and the beam size of the observations at the two frequencies, if some filaments are resolved.
}

\section{Discussion: the origin of the molecular gas near M 86}

Li \& van Gorkom (2001) attributed the origin of the H I emission, which peaks near CO-emitting regions, to gas that cooled down from a compressed, shocked, and heated medium. In cooling flows, however, the ICM gas is depleted of its dust due to sputtering by X-rays. Dust sputtering leads to gas-to-dust ratios that exceed their typical values for local galaxies (i.e. several hundreds; Young \& Scoville 1991; Wiklind et al. 1995) in timescales of only Myr in $10^{6}-10^{7} \mathrm{~K}$ environments (Clemens et al. 2010). On the other hand, the presence of dust in all M 86 regions where $\mathrm{CO}$ was detected (Figs. 3 and 4; Cortese et al. 2010b; Gomez et al. 2010) indicates that the gas and the dust were commonly accumulated. Their relative mass ratio indeed favors the capture from an external galaxy rather than the formation from a cooling ICM. For the inner $2^{\prime}$ of M 86, the total $15-20 \mathrm{~K}$ dust mass is $(2-5) \times 10^{6} M_{\odot}$ (Gomez et al. 2010). If the molecular gas distribution follows the dust distribution, most of the $\mathrm{H}_{2}$ gas in the inner $2^{\prime}$ will be accounted for by the observations at V6, V3NW, V3W, V3WW, V8, M86SW, and at the center of $\mathrm{M} 86$. The total $\mathrm{H}_{2}$ mass will then be in the range (2-5) $\times 10^{7} M_{\odot}$. Furthermore assuming that the center of M 86 comprises $1.1 \times 10^{7} M_{\odot}$ of atomic gas based on its $\mathrm{H} \alpha+[\mathrm{N} \mathrm{II}]$ luminosity (Kenney et al. 2008), and another $5.6 \times 10^{7} M_{\odot}$ based on its H I observations (Bregman \& Roberts 1990), we deduct that its gas-to-dust ratio will be in the range $20-60$. The range can be broader when considering the different beam sizes of the observations. For comparison, Cortese et al. (2010b) give a 10-20 K dust mass of $2 \times 10^{6}-2 \times 10^{7} M_{\odot}$, leading to a gas-to-dust ratio of 30-300 (including helium) for a dust knot $5 \mathrm{kpc}$ northwest of NGC 4438.

With its $\mathrm{H} \alpha$-emitting trail, NGC 4438 is the best candidate for the external capture of the gas and the dust in M 86. While the trail started elongating as the two galaxies were receding from each other, most of the gas and the dust was likely captured when the galaxies where near the pericenter of their encounter. If gas was transferred from NGC 4438 to M 86 in ionized or neutral state through dynamical instabilities or ISM-ISM stripping, CO could be detected in M 86 if it reformed in situ. Molecular formation is possible to occur in situ in the V3W and V3WW complexes. The column density threshold for the efficient conversion of $\mathrm{HI}$ to $\mathrm{H}_{2}$ is in the range $3 \times 10^{20}-10^{21} \mathrm{~cm}^{-2}$ (Schaye 2004). The $\mathrm{HI}$-deduced column density in these regions is close to the $1.6 \times 10^{20} \mathrm{~cm}^{-2}$ contour of Kenney et al. (2008). Likewise, the CO-based $\mathrm{H}_{2}$ column density is $2.9 \times 10^{20}$ and $1.9 \times 10^{20} \mathrm{~cm}^{-2}$ for V3W and V3WW, respectively, for the adopted CO-to- $\mathrm{H}_{2}$ Galactic conversion factor. The $\mathrm{H}$ I column density requirement would be satisfied if the $\mathrm{H}$ I-to- $\mathrm{H}_{2}$ mass ratio was $\gtrsim 3$, which is comparable to that of both moderately stripped spirals and ellipticals (Kenney \& Young 1989; Wiklind et al. 1995). The present computations assume that the gas emission is smeared out across each telescope's beam. When taking into account the clumpy distribution of the clouds, the column density requirement can be easily met. For this scenario to hold, the CO needs to be detected in regions where dust is also present, because the dust particles act like catalysts for the H I atoms to recombine upon. This condition is also satisfied.

Contrarily to the $\mathrm{V} 3 \mathrm{~W}(\mathrm{~W})$ complexes, the bulk of the molecules could not have formed in situ in V7, where the $\mathrm{HI}_{\mathrm{I}}$ deduced column density is outside the last contour of Kenney et al. (2008), i.e., below $10^{19} \mathrm{~cm}^{-2}$. The detection of CO there provides evidence for the survival and transport of self-shielded molecular clouds in the hot ICM of Virgo. The molecules could 


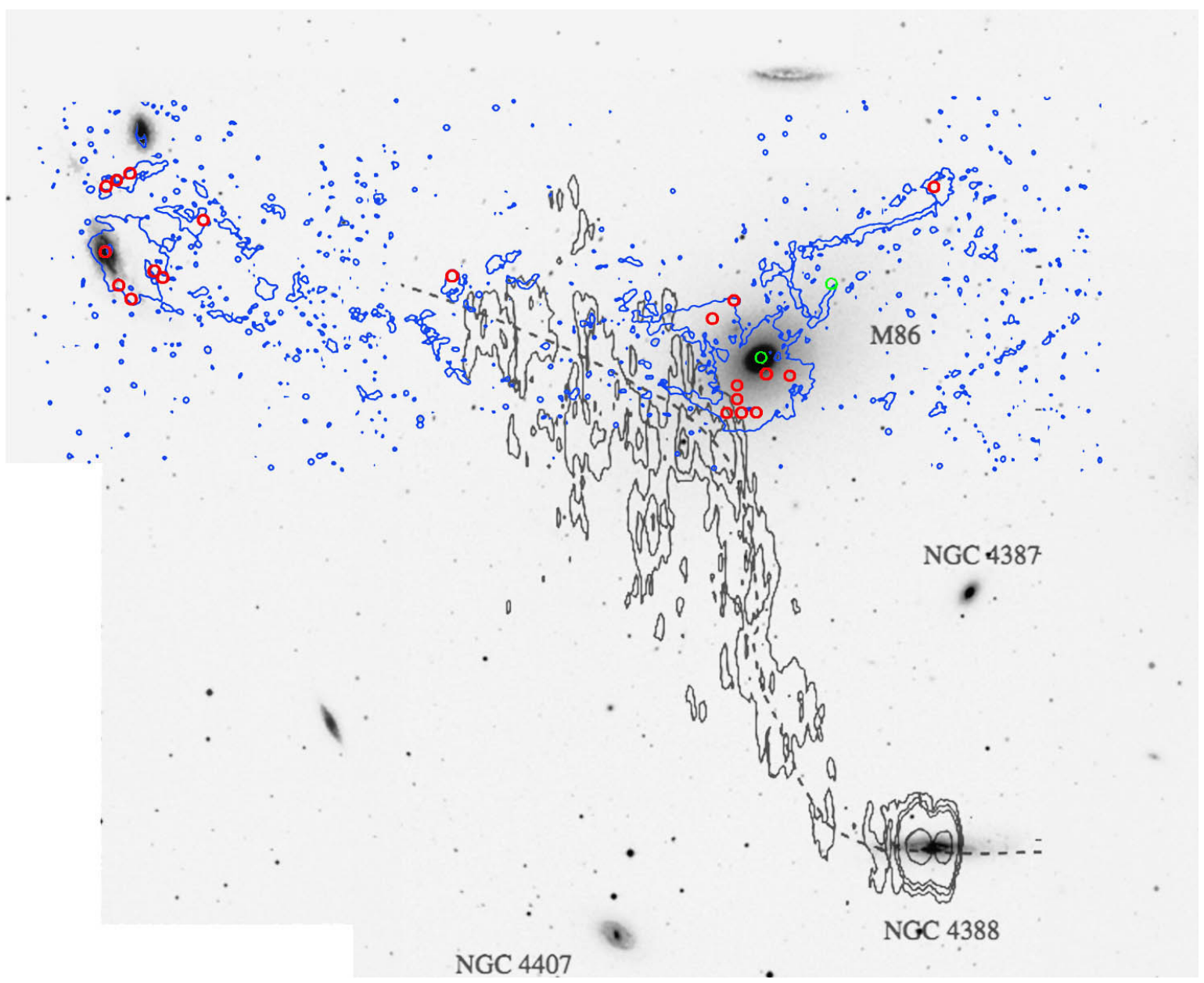

Fig. 5. CO observation positions and $\mathrm{H} \alpha$ intensity contours (as in Fig. 3) and H I intensity contours (black; Oosterloo \& van Gorkom 2005) plotted over a DSS $B$-band image.

have started forming, e.g., near the Hi peak south of M 86 and then rotated around it in a half-circular orbit of $\sim 30 \mathrm{kpc}$. If the clouds were moving at $300 \mathrm{~km} \mathrm{~s}^{-1}$ (i.e. close to the V3W and V7 difference in recession velocity), their travel time would be 100 Myr. Because the formation of new stars from $\mathrm{H}_{2}$ in isolated clouds takes place in timescales of $10^{5}-10^{7} \mathrm{yr}$ (e.g., Krumholtz et al. 2012), this result implies that the collapse, the fragmentation, and the reformation of the molecular clouds from gas in their vicinity is a continuous process inside filaments.

Alternatively, gas clumps could have been transferred from NGC 4438 to M 86 directly in a dense, molecular state. The molecular gas has then survived near M 86 for a time comparable to the travel time of NGC 4438 from the pericenter to its present location. The orbital simulations of Vollmer et al. (2009) also suggest that NGC 4438 has been moving through the hot X-ray gas for over only $100 \mathrm{Myr}$ after its encounter with M 86. This timescale is insufficient for the various energy/momentum transport processes that act on the molecular clouds to fully destroy them. These processes include the heat conduction from the surrounding medium, the viscous flow stripping, and the Kelvin-Helmholtz instabilities, with the heat conduction leading to the highest mass loss rates in this environment (Nulsen 1982). In the ICM, the mean free path of the electrons is large compared to the radii of the molecular clouds, leading to saturated heat conduction and long evaporation timescales (Cowie \& McKee 1977). For a cloud with radius of $10 \mathrm{pc}$ and density $n$ of $10^{3} \mathrm{~cm}^{-3}$ that is embedded in a $10^{7} \mathrm{~K}$ medium with density of $10^{-4} \mathrm{~cm}^{-3}$, the evaporation time is $3 \mathrm{Gyr}$. This time can increase when magnetic fields and radiative cooling are taken into account (Cowie \& McKee 1977; McKee \& Cowie 1977), because cooling is very efficient above $10^{4} \mathrm{~K}$ (Dalgarno \& McCray 1972). It effectively dominates over heating for clouds that are stripped outside of galaxies and that are not sufficiently heated by stellar ultraviolet radiation (Vollmer et al. 2001). The atomic gas will then cool down, condense, and reform $\mathrm{H}_{2}$ within $\sim 10^{9} / n \mathrm{yr} \mathrm{cm}^{-3}$ (Hollenbach \& Tielens 1997). It is therefore plausible that the molecular gas observed in M 86 was directly stripped from NGC 4438, even though it was not detected in our observations of, e.g., the V1 region. This might occur if there is no strong source of gas excitation, such as star formation, shocks, cosmic rays (Ferland et al. 2008), or diffuse intracluster light (Mihos et al. 2005).

An entirely different scenario that remains to be tested is whether the gas in M 86 could originate from NGC 4388, a spiral galaxy at $\sim 100 \mathrm{kpc}$ in projection on its south, with an estimated $\mathrm{H}_{2}$ reservoir of $10^{8}-10^{9} M_{\odot}$ (Kenney \& Young 1989; Papadopoulos \& Seaquist 1998). NGC 4388 could be interacting with the M 86-NGC 4438 complex, if all galaxies were at a sufficiently close distance (e.g., Willick et al. 1997). The interaction scenario was considered after the detection of a long $(>120 \mathrm{kpc})$ trail of neutral gas starting from NGC 4388 and heading in the direction of M 86 and NGC 4438 (Oosterloo \& van Gorkom 2005, Fig. 5), and by the strong gas stripping in this system (e.g., Kenney \& Young 1989; Vollmer et al. 2009). Very Large Array 


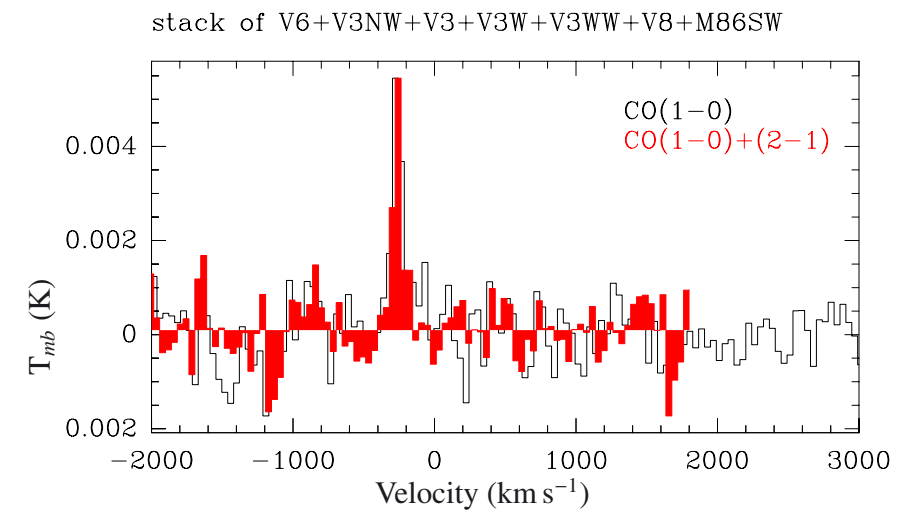

Fig. 6. Stacked spectra of all positions south of M 86. No gas is detected at any velocity other than the galaxy's recession velocity.

data indicated that NGC 4388 has a radio jet that is driven by an active nucleus (Colbert et al. 1996; Irwin et al. 2000) and that could be adding to the gas expulsion mechanisms. A massive outflow has been observed in its $\mathrm{H} \alpha$ and [ $\mathrm{N}_{\text {III }}$ images (Veilleux et al. 1999; Kenney et al. 2008), with an $\mathrm{H} \alpha$ velocity ranging between $2000 \mathrm{~km} \mathrm{~s}^{-1}$ and $2400 \mathrm{~km} \mathrm{~s}^{-1}$ (Yoshida et al. 2004). The velocity of the neutral, $\mathrm{HI}$ I-emitting gas along the trail is likewise between 2000-2700 $\mathrm{km} \mathrm{s}^{-1}$ (Cayatte et al. 1990; Oosterloo $\&$ van Gorkom 2005; Chung et al. 2009). The projected H I detection limits are close to the $\mathrm{H} \alpha$ detection limits in the vicinity of M 86 (Fig. 5). However, the non-detection of $\mathrm{CO}$ moving at any velocity between $-250 \mathrm{~km} \mathrm{~s}^{-1}$ and $2700 \mathrm{~km} \mathrm{~s}^{-1}$ in the south of M 86 (either in individual or stacked spectra; Fig. 6) renders this scenario improbable. A similar conclusion was drawn by Oosterloo \& van Gorkom (2005) based on the H I gas kinematics. Additional (and deeper) observations would be needed to test the viability of this scenario.

\section{Summary}

Using IRAM's $30 \mathrm{~m}$ telescope, we investigated the presence of molecular gas in Virgo's hot ICM, and specifically in the vicinity of M 86, NGC 4438, and in the $120 \mathrm{kpc}$-long tidal trail that connects them. We focused on $\mathrm{H} \alpha$ emission peaks, which could originate from the interface of dense clouds with self-shielded CO molecules and the X-ray emitting ICM, because ram pressure stripping has dispersed most of the diffuse ionized gas. We observed 21 regions in the ${ }^{12} \mathrm{CO}(1-0)$ and ${ }^{12} \mathrm{CO}(2-1)$ transitions and draw the following main conclusions.

- Narrow CO emission, with line widths of only $1.2 \mathrm{~km} \mathrm{~s}^{-1}$, was detected at 12:27:30+13:12:12, northwest of NGC 4438 and NGC 4435. It is tracing Galactic cirrus clouds. In no other position were the $\mathrm{CO}$ line widths characteristic of those in the cirrus.

- CO was detected at more than $10 \mathrm{kpc}$ away from the centers of NGC 4438 and M 86. For example, strong CO emission was found in the tidal structure north of NGC 4438, in the regions E2E, E2, and E2W. Through these cloud complexes, $5 \times 10^{7} M_{\odot}$ or $9 \%$ of the gas in the center of NGC 4438 could be lost to the ICM and potentially to neighboring galaxies.

- CO was discovered in an $\mathrm{H} \alpha$ stream that ends in the center of M 86, at $\sim 10 \mathrm{kpc}$ southeast from the center, and near the peak of the $\mathrm{HI}$ emission. The $\mathrm{CO}$ recession velocity agrees well with that of the $\mathrm{H}_{\mathrm{I}}$ and the stars. The ${ }^{12} \mathrm{CO}(1-0)$ line properties indicate the presence of $2 \times 10^{7} M_{\odot}$ of $\mathrm{H}_{2}$ in the combined regions V3W and V3WW. Some of this gas could have formed in situ given that the local $\mathrm{H}$ I surface brightness exceeds $10^{20} \mathrm{~cm}^{-2}$.

- The filamentary structure $11 \mathrm{kpc}$ northeast from the center of $\mathrm{M} 86$, in the region that is denoted $\mathrm{V7}$, contains $\mathrm{CO}$ moving at $-530 \mathrm{~km} \mathrm{~s}^{-1}$. Its corresponding $\mathrm{H}_{2}$ mass is $7 \times 10^{6} M_{\odot}$. Because there is no associated $\mathrm{H}_{\mathrm{I}}$ emission in this region or in its vicinity, we attribute this $\mathrm{CO}$ detection to gas that was primarily transferred to V7 in molecular form. We interpret it as survival of self-shielded, re-forming molecular clouds in filaments for $\sim 100$ Myr. This is longer than the fragmentation timescale of individual isolated clouds, and shorter than the evaporation timescale due to heat conduction from the ICM.

- The integrated gas-to-dust ratio in the inner $2^{\prime}$ of M 86, $20-60$, is too low to be consistent with gas cooling from the ICM. The molecular gas detected in and near M 86 is therefore likely to have originated from an external galaxy.

- The most probable scenario for the molecular gas origin of M 86 is that it comes from NGC 4438. The best evidence for this remains the smooth $\mathrm{H} \alpha$ velocity gradient in the bridge between them, combined with the similar $\mathrm{H} \alpha$ and $\mathrm{CO}$ velocities, where detected.

- The scenario of gas being transferred to M 86 from NGC 4388 on its south was also examined, given the $\mathrm{H}_{\mathrm{I}}$ stream that is seen in projection between these galaxies. However, the non-detection of $\mathrm{CO}$ below or in the observed range of Hi recession velocities, i.e., between 2000 $2700 \mathrm{~km} \mathrm{~s}^{-1}$, does not favor this scenario.

Acknowledgements. K.D. acknowledges support by the Centre National d'Études Spatiales (CNES). We are thankful to G. Novak for constructive discussions, to the HeVICS team for making their data publicly available, and to the anonymous referee who helped us to improve this document. Based on observations carried out with the IRAM $30 \mathrm{~m}$ telescope. IRAM is supported by INSU/CNRS (France), MPG (Germany) and IGN (Spain).

\section{References}

Bender, R., Burstein, D., \& Faber, S. M. 1992, ApJ, 399, 462

Bloemen, J. B. G. M., Strong, A. W., Mayer-Hasselwander, H. A., et al. 1986, A\&A, 154, 25

Braine, J., Henkel, C., \& Wiklind, T. 1997, A\&A, 321, 765

Braine, J., Lisenfeld, U., Duc, P.-A., \& Leon, S. 2000, Nature, 403, 867

Bregman, J. N., \& Roberts, M. S. 1990, ApJ, 362, 468

Cayatte, V., van Gorkom, J. H., Balkowski, C., \& Kotanyi, C. 1990, AJ, 100, 604

Chung, A., van Gorkom, J. H., Kenney, J. D. P., Crowl, H., \& Vollmer, B. 2009, AJ, 138, 1741

Clemens, M. S., Jones, A. P., Bressan, A., et al. 2010, A\&A, 518, L50

Cluver, M. E., Appleton, P., Boulanger, F., et al. 2010, ApJ, 710, 248

Colbert, E. J. M., Baum, S. A., Gallimore, J. F., O'Dea, C. P., \& Christensen, J. A. 1996, ApJ, 467, 551

Combes, F., Dupraz, C., Casoli, F., \& Pagani, L. 1988, A\&A, 203, L9

Conselice, C. J., Gallagher, J. S., III, \& Wyse, R. F. G. 2001, AJ, 122, 2281

Cortese, L., Bendo, G. J., Isaak, K. G., et al. 2010a, MNRAS, 403, L26

Cortese, L., Bendo, G. J., Boselli, A., et al. 2010b, A\&A, 518, L63

Cowie, L. L., \& McKee, C. F. 1977, ApJ, 211, 135

Cowie, L. L., Hu, E. M., Jenkins, E. B., \& York, D. G. 1983, ApJ, 272, 29

Crowl, H. H., Kenney, J. D. P., van Gorkom, J. H., \& Vollmer, B. 2005, AJ, 130, 65

Dalgarno, A., \& McCray, R. A. 1972, ARA\&A, 10, 375

Davies, J. I., Baes, M., \& Bendo, G. J., et al. 2010, A\&A, 518, L48

Downes, D., Solomon, P. M., \& Radford, S. J. E. 1993, ApJ, 414, L13

Fabian, A. C. 1994, ARA\&A, 32, 277

Fabian, A. C., Sanders, J. S., Taylor, G. B., et al. 2006, MNRAS, 366, 417

Ferland, G. J., Fabian, A. C., Hatch, N. A., et al. 2008, MNRAS, 386, L72

Ferland, G. J., Fabian, A. C., Hatch, N. A., et al. 2009, MNRAS, 392, 1475

Forman, W., Schwarz, J., Jones, C., Liller, W., \& Fabian, A. C. 1979, ApJ, 234, L27

Gao, Y., \& Solomon, P. M. 2004, ApJ, 606, 271 
K. M. Dasyra et al.: CO in Virgo's hot intracluster medium

Gavazzi, G., Donati, A., Cucciati, O, et al. 2005, A\&A, 430, 411

Gomez, H. L., Baes, M., Cortese, L., et al. 2010, A\&A, 518, L45

Gunn, J. E., \& Gott, J. R. III 1972, ApJ, 176, 1

Heckman, T. M., Baum, S. A., van Breugel, W. J. M., \& McCarthy, P. 1989, ApJ, 338,48

Hollenbach, D. J., \& Tielens, A. G. G. M. 1997, ARA\& A, 35, 179

Hota, A., Saikia, D. J., \& Irwin, J. A. 2007, MNRAS, 380, 1009

Irwin, J. A., Saikia, D. J., \& English, J. 2000, AJ, 119, 1592

Johnstone, R. M., Hatch, N. A., Ferland, G. J., et al. 2007, MNRAS, 382, 1246

Kenney, J. D. P., \& Young, J. S. 1989, ApJ, 344, 171

Kenney, J. D. P., Tal, T., Crowl, H. H., et al. 2008, ApJ, 687, L69

Kennicutt, R. C., Jr. 1998, ARA\&A, 36, 189

Krumholz, M. R., Dekel, A., \& McKee, C. F. 2012, ApJ, 745, 69

Li, Y., \& van Gorkom, J. 2001, ASP Conf., 240, 637

Liu, J.-F., \& Bregman, J. N. 2005, ApJS, 157, 59

O’Sullivan, E. O., Giacintucci, S., Vrtilek, J. M., Raychaudhury, S., \& David, L. P. 2009, ApJ, 701, 1560

Oosterloo, T., \& van Gorkom, J. 2005, A\&A, 437, L19

Machacek, M. E., Jones, C., \& Forman, W. R. 2004, ApJ, 610, 183

Mahdavi, A., Margaret, J., Fabricant, D. G., et al. 1996, AJ, 111, 1

McKee, C. F., \& Cowie, L. L. 1977, ApJ, 215, 213

Mei, S., Blakeslee, J. P., Coté, P., et al. 2007, ApJ, 655, 144

Mihos, J. C., Harding, P., Feldmeier, J., \& Morrison, H. 2005, ApJ, 631, L41

Nulsen, P. E. J. 1982, MNRAS, 198, 1007

Papadopoulos, P. P., \& Seaquist, E. R. 1998, ApJ, 492, 521
Randall, S., Nulsen, P., Forman, W. R., et al. 2008, ApJ, 688, 208

Rangarajan, F. V. N., White, D. A., Ebeling, H., \& Fabian, A. C. 1995, MNRAS, 277,1047

Salomé, P., Combes, F., Edge, A. C., et al. 2006, A\&A, 454, 437

Salomé, P., Combes, F., Revaz, Y., et al. 2011, A\&A, 531, A85

Schaye, J. 2004, ApJ, 609, 667

Solomon, P. M., Downes, D., Radford, S. J. E., \& Barrett, J. W. 1997, ApJ, 478, 144

Sivanandam, S., Rieke, M. J., \& Rieke, G. H. 2010, ApJ, 717, 147

Smith, R. J., Lucey, J. R., Hudson, M. J., Schlegel, D. J., \& Davies, R. L. 2000, MNRAS, 313, 469

Sun, M., Donahue, M., Roediger, E., et al. 2010, ApJ, 708, 946

Tamura, T., Maeda, Y., Mitsuda, K., et al. 2009, ApJ, 705, L62

Trinchieri, G., Sulentic, J., Pietsch, W., \& Breitschwerdt, D. 2005, A\&A, 444, 697

Veilleux, S., Bland-Hawthorn, J., Cecil, G., Tully, R. B., \& Miller, S. T. 1999, ApJ, 520, 11

Vollmer, B., Cayatte, V., Balkowski, C., \& Duschl, W. J. 2001, ApJ, 561, 708

Vollmer, B., Braine, J., Combes, F., \& Sofue, Y. 2005, A\&A, 441, 473

Vollmer, B., Braine, J., Pappalardo, C., \& Hily-Blan, P. 2008, A\&A, 491, 455

Vollmer, B., Soida, M., Chung, A., et al. 2009, A\&A, 496, 669

Willick, J. A., Courteau, S., Faber, S. M., et al. 1997, ApJS, 109, 333

Wiklind, T., Combes, F., \& Henkel, C. 1995, A\&A, 297, 643

Yoshida, M., Ohyama, Y., Iye, M., et al. 2004, AJ, 127, 90

Young, J. S., \& Scoville, N. Z. 1991, ARA\&A, 29, 581 